\title{
CLASSIFICADOR TEXTURAL PARA IDENTIFICAÇÃO DE PLANTAS
}

José Antonio Rodrigues de Souza ${ }^{1}$, Débora Astoni Moreira ${ }^{2}$

\section{RESUMO}

No presente trabalho, objetivou-se desenvolver um classificador textural em Matlab, para identificação de quatro espécies de plantas. A textura foi definida por meio de um conjunto de medidas estatísticas, descrevendo as variações espaciais de intensidade de pixels ou cor da parte aérea das plantas. As medidas foram calculadas utilizando-se matrizes de coocorrência. Os resultados indicaram que a classificação textural para identificação de plantas é uma das mais simples possíveis, sendo os resultados das classificações extremamente relacionados com as texturas analisadas.

Palavras-chave: classificação de imagens, textura, matriz de co-ocorrência

\section{TEXTURE CLASSIFIER FOR PLANT IDENTIFICATION}

\begin{abstract}
The objective of this work was to develop a texture classifier in Matlab to identify four plant species. The texture was defined by means of a set of statistical measures describing the spatial variations of pixel intensity or color of the shoots. Such measures were calculated using matrices of co-occurrence. The results showed that the textural classification for plant identification is simple, because the classification results highly related to the analyzed textures.
\end{abstract}

Keywords: classification of images, texture, matrix of co-occurrence

\section{Recebido para publicação em 24/08/2009. Aprovado em 11/11/2009}

1- Prof. IFET - GO, Rodovia Geraldo da Silva Nascimento, km 2,5, Urutaí-GO, email: jarstec@yahoo.com.br.

2- Prof'. UEMG - Ubá-MG, Rua: Olegário Maciel, 1427- Bairro Industrial - Ubá - MG, email: deboraastoni@yahoo.com.br 


\section{INTRODUÇÃO}

A textura é uma das características mais importantes para classificar e reconhecer objetos e cenas, podendo ser caracterizada por variações locais, em valores de pixels, que se repetem de maneira regular ou aleatória, ao longo do objeto ou da imagem. Vários métodos para classificação de imagens utilizando características de textura têm sido propostos na literatura (MARCEAU, 1990; LIEW, 1995; SOLBERG e JAIN, 1995; LIVARINEN e VISA, 1998; MUDIGONDA et al., 2000; PIERCE, 1998; SHABAN e DIKSHIT, 1998; SCHOWENGERDT, 2000; e AYALA e DOMINGO, 2001). Entretanto, não há um método genérico ou uma abordagem formal que seja útil para uma grande variedade de imagens (AKSOY, 1998).

As três abordagens principais usadas em classificação de imagens para a descrição de texturas são a estatística, a estrutural e a espectral. $\mathrm{Na}$ abordagem estatística, a textura é definida por um conjunto de medidas locais, extraídas do padrão. Medidas estatísticas comuns incluem entropia, correlação, contraste e variância.

A matriz de coocorrência (MC) é uma tabulação de quantas combinações diferentes de valores de intensidade dos pixels (níveis de cinza) ocorrem em uma imagem. A idéia principal da MC é caracterizar texturas em uma imagem por meio de um conjunto de estatísticas para as ocorrências de cada nível de cinza em pixels diferentes ao longo de diferentes direções.

Co-ocorrência, na sua forma geral, pode ser especificada por uma matriz de frequências relativas $P(i, j ; d, \theta)$, na qual dois elementos de textura vizinhos, separados por uma distância $\mathrm{d}$ em uma orientação $\theta$, ocorrem na imagem, um com propriedade $\mathrm{i}$ e o outro com propriedade $\mathrm{j}$. Instanciando essa definição para co-ocorrência de níveis de cinza, os elementos de textura são pixels e as propriedades são os níveis de cinza. Por exemplo, para um relacionamento com um ângulo $\theta=0^{\circ}, \mathrm{P}$ $(\mathrm{i}, \mathrm{j} ; \mathrm{d}, \theta$ ) é a média aritmética da probabilidade de uma transição, da esquerda para a direita, do nível de cinza i para o nível de cinza j, com uma distância d. Essas matrizes podem ser normalizadas por meio da divisão de cada entrada da matriz pelo número de pixels vizinhos usados para calcular aquela matriz (AKSOY, 1998).

Com o objetivo de utilizar a informação contida nas matrizes de co-ocorrência de níveis de cinza são utilizadas medidas estatísticas para medir características texturais (HARALICK et al., 1973) como momento angular, variância, média e entropia.

Essa parte da introdução está muito didática. Deve ser reescrita.

- Momento angular: é uma medida da homogeneidade da imagem. Poucas mudanças de intensidade acarretam poucos valores de alta magnitude na matriz de coocorrência, resultando num alto momento angular. Valores elevados ocorrem quando a imagem está muito ordenada. $\mathrm{O}$ momento angular pode ser determinado pela seguinte expressão:

$$
C_{1}=\sum_{i=1}^{L} \sum_{j=1}^{L}[p(i j)]^{2}
$$

em que,

C1 - momento angular; p(ij) - elementos da matriz de coocorrência.

- Variância: quando utilizada na textura, executa a mesma tarefa que a variância utilizada na estatística descritiva. Baseia-se na dispersão dos dados numéricos em torno de um valor médio, de valores dentro da MC. Entretanto, a variância da $\mathrm{MC}$ trata especificamente das combinações do pixel de referência e do seu vizinho. Então, isso não é o mesmo que a simples variância de níveis de cinza na imagem original.

$$
C_{3}=\sum_{i=1}^{L}\left(i-C_{2}\right)^{2} p_{x}(i)
$$

em que,

$\mathrm{C}_{3}$ - variância; i - valor observado; $\mathrm{C}_{2}$ - média; $P_{x}(i)$ - frequência do valor observado.

- Média: é uma medida do brilho da imagem, representado em uma matriz de co-ocorrência, e pode ser determinada pela seguinte expressão:

$$
C_{2}=\sum_{i=1}^{L} i^{*} p_{x}(i)
$$


em que,

$\mathrm{C}_{2}$ - média; $\mathrm{P}_{\mathrm{x}}$ (i) - frequência do valor observado.

- Entropia: a entropia mede a desordem em uma imagem, ou seja, o grau de dispersão de níveis de cinza, e é determinada pela seguinte expressão:

$$
C_{4}=\sum_{i=1}^{L} \sum_{j=1}^{L} \mathrm{p}(\mathrm{i}, \mathrm{j}) \ln \mathrm{p}(\mathrm{i}, \mathrm{j})
$$

em que,

C4 - entropia; p (i,j) - matriz de coocorrência; ln logaritmo neperiano.

Um Classificador Bayesiano é um método probabilístico aplicado à tarefa de classificação. Esses classificadores aprendem a partir dos exemplos de treinamento e geram uma estrutura de representação, com base no cálculo de probabilidades condicionais. São utilizados para determinar a probabilidade de um novo exemplo pertencer a uma dada classe, considerando-se os valores de seus atributose utilizando-se as probabilidades estabelecidas para o conjunto de treinamento (DUDA e HART, 1973).

O método Bayesiano é uma forma de estabelecer regras de decisão. As amostras de treinamento servem para estabelecer a distribuição de probabilidade de cada uma das classes. Cada classe $W_{k}$ é modelada por uma função densidade de probabilidade $p\left(X \mid W_{k}\right)$ do tipo gaussiana. Um determinado pixel da imagem com valor espectral $X=X_{0}$ deve ser associado à classe mais provável, isto é, àquela que maximize $P\left(W_{k} \mid X_{0}\right)$, que é a probabilidade de que a classe de $X_{0}$ seja $W_{k}$. Intuitivamente, cada classe está associada a uma curva de distribuição de probabilidade; dependendo da posição espectral do pixel em relação a essas curvas, é determinada a classe a que ele pertence. Segundo a regra de Bayes:

$$
P\left(W_{k} \mid X\right)=\frac{P\left(W_{k} \mid X\right) P\left(W_{k}\right)}{P(X)}
$$

O valor $P(X)$ é independente das classes, logo, basta maximizar o numerador de (5). Supondose que, inicialmente, as classes tenham igual probabilidade, ou seja, $P\left(W_{l}\right)=P\left(W_{2}\right)=\ldots=$
$P\left(W_{j}\right)$, temos que o único termo a ser maximizado é $P\left(X \mid W_{k}\right)$, que, segundo o modelo gaussiano, tem a seguinte expressão:

$P\left(W_{k} \mid X\right)=\frac{1}{(2 \pi)^{1 / 2}\left|C_{k}\right|^{1 / 2}} \exp \left(\frac{1}{2}\left(X-M_{k}\right)^{t} C_{k}\left(X-M_{k}\right)\right.$

em que,

n - número de atributos do pixel (número de bandas espectrais da imagem); $\mathrm{M}_{\mathrm{k}}$ - vetor-média da classe $W k ; \mathrm{C}_{\mathrm{k}}$ - matriz de covariância da classe $W k$.

No presente trabalho, objetivou-se desenvolver um classificador textural, em Matlab, para identificação de plantas.

\section{MATERIAL E MÉTODOS}

O trabalho foi realizado no Departamento de Engenharia Agrícola da Universidade Federal de Viçosa, Viçosa, Minas Gerais. Neste estudo utilizaram-se quatro espécies de plantas (Leiteiro - Euphorbia heterophylla, Colchão - Digitaria horizontalis, Milho - Zea mays e Timbete Cenchrus echinatus), com três imagens de cada espécie, totalizando 12 imagens analisadas (Figura 1).

Para a análise das imagens, empregouse a estatística de segunda ordem, baseada no Método da Diferença de Nível de Cinza (GLDM-Grey Level Difference Method), sendo calculadas as características de cada espécie de planta por meio de matrizes de coocorrência, conforme Haralick et al. (1973). Optou-se por este método em virtude de apresentar melhor eficiência na avaliação de conjunto de texturas (CONNERS e HARLOW, 1980) e pelo fato de as texturas a serem analisadas não serem repetitivas, não apresentando, portanto, boa regularidade (TURCEYAN e JAIN, 1993; LI, 1995). A estatística de segunda ordem foi calculada pela probabilidade de um determinado par de níveis de cinza $i$ e $j$, numa determinada distância $\delta$ e uma direção $\theta$, ocorrer, ou seja, a GLDM pode ser denominada como sendo um histograma bidimensional que fornece a frequência de ocorrência $P(i, j, \delta, \theta)$ (FERREIRA e FIGUEIREDO, 2006). 


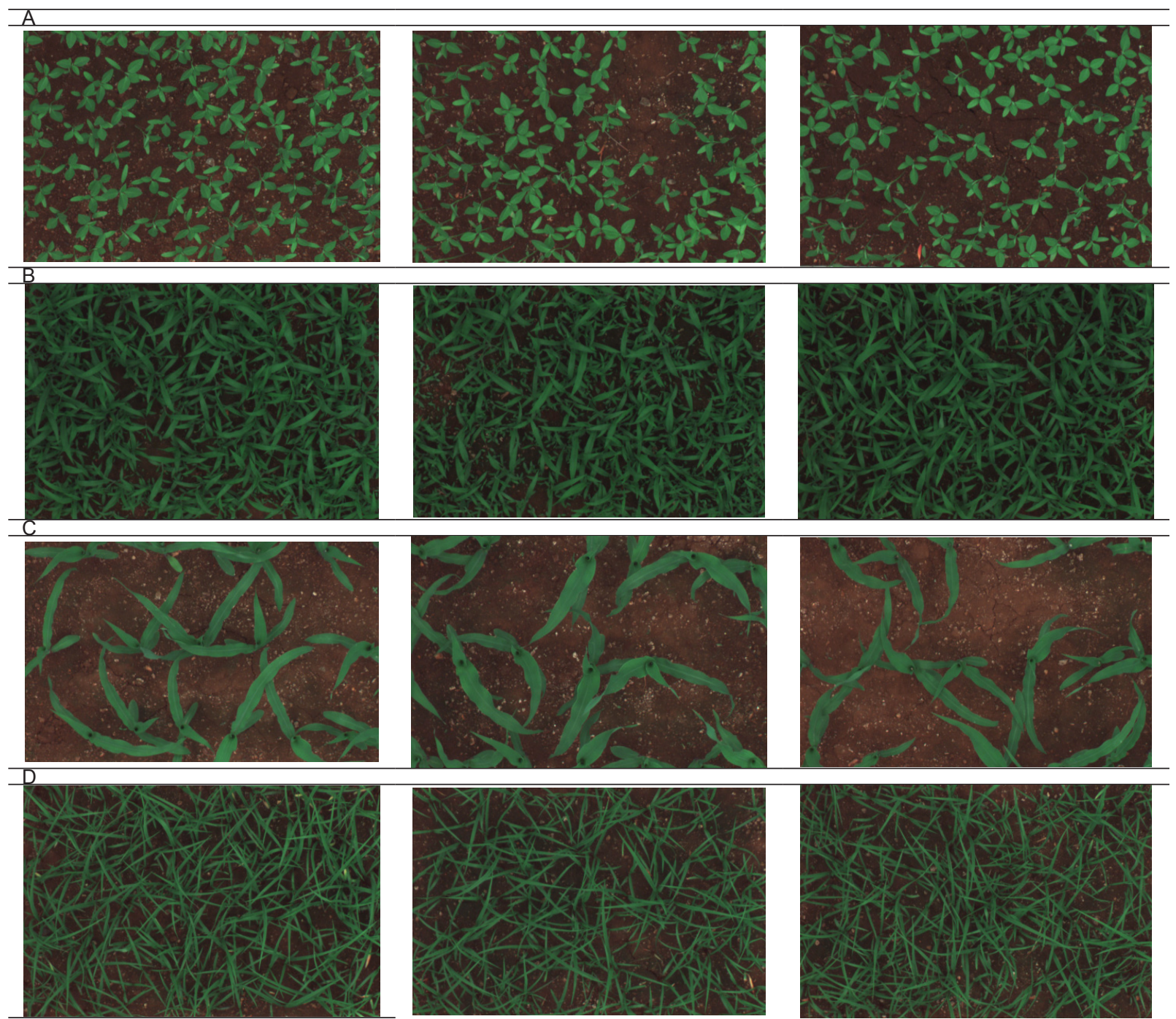

Figura 1. Espécies de plantas utilizadas para o desenvolvimento do classificador textural: Leiteiro (A), Colchão (B), Milho (C) e Timbete (D).

A abordagem, utilizando características de textura para identificar as diferentes plantas, envolveu três etapas principais: inicialização, treinamento e classificação.

Na fase de inicialização, ocorreu a quantização da imagem colorida original (RGB) para uma imagem monocromática, utilizando-se 256 níveis de cinza.

$\mathrm{Na}$ fase de treinamento, selecionaram-se, aleatoriamente, cinco imagens de cada espécie de planta, retirando-se cinco matrizes de dimensões 70 x 70 pixels. Para cada espécie analisada, calcularamse as quatro matrizes de coocorrência: horizontal $\left(0^{\circ}\right)$, vertical $\left(90^{\circ}\right)$, diagonal direita $\left(45^{\circ}\right)$ e diagonal esquerda $\left(135^{\circ}\right)$. A seguir, essas matrizes foram normalizadas, a fim de se determinar as matrizes de probabilidade.

A partir da matriz de probabilidade foram calculadas as estatísticas de segunda ordem, ou seja, os descritores estruturais de interesse: (momento angular, valor médio, variância e entropia). O processo de cálculos dos descritores estruturais foi feito para cada espécie de planta, montando-se o vetor de características.

Na fase de classificação, para agilizar o processo, criou-se uma imagem completa, resultante da montagem, em uma única imagem, da sequência das três imagens para as quatro espécies de plantas analisadas. Nesta fase, utilizaram-se o Classificador Bayesiano para Classes Gaussianas (CGB), sendo que o processo descrito na Figura 2 foi realizado percorrendo-se toda a imagem completa, para cada matriz de 70 x 70 pixels. A partir do valor encontrado, compararam-se as sequências?, por meio do vetor de características, para que, assim, o conjunto de pixels fosse classificado de acordo com a classe de que mais se aproximava. 


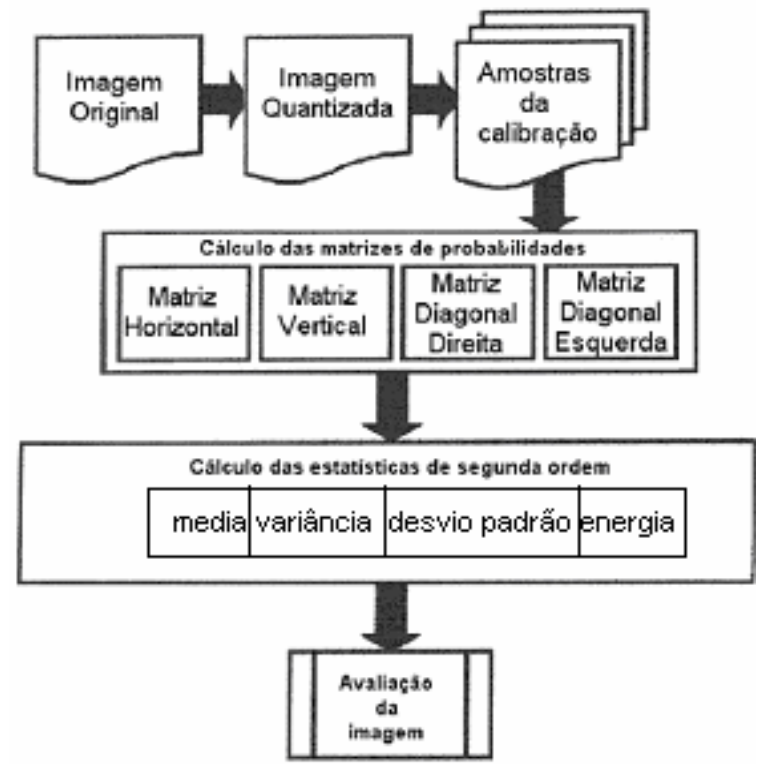

Figura 2. Esquema das etapas realizadas na classificação das plantas

Para visualização dos resultados, após a classificação foram criadas novas imagens, contendo quatro diferentes níveis de cinza (60 - Leiteiro, 120 - Colchão, 180 - Milho e 240 - Timbete). Assim, após classificação, cada conjunto de matriz analisada recebia um nível de cinza diferente, referente à espécie que o classificador reconhecia e classificava nessa matriz.

Para verificação da eficiência de classificação das quatro direções analisadas, determinou-se o índice Kappa que, de acordo com Congalton et al. (1993), é um dos parâmetros mais utilizados e eficientes na determinação da exatidão, sendo obtido com determinação da matriz de erro.

A matriz de erro ou matriz de confusão é uma matriz quadrada, de números que expressam a quantidade de unidades amostrais, associada a uma dada categoria durante o processo de classificação efetuado, e a categoria real a que pertencem essas unidades, de acordo com os autores: Rosenfield e Fitzpatrick (1986); Story (1986) e Congalton (1993). A matriz de erro favorece a visualização dos resultados da classificação temática e expressa a relação entre os dois tipos de erros associados ao sistema de classificação: os de inclusão e os de omissão (CARD, 1982).

O índice Kappa mede a concordância entre diferentes medidas, métodos ou instrumentos de medição, com os seguintes critérios: abaixo de 0,40 , baixa concordância; entre 0,40 e 0,75 , concordância razoável e acima de 0,75, excelente concordância (PEREIRA et al., 2000). O coeficiente Kappa é calculado a partir da Equação 7 (BISHOP et al., 1975).

$$
\hat{K}=\frac{N \sum_{i=1} X_{i \ddot{i}}-\sum_{i=1}\left(X_{i+} * X_{+i}\right)}{N^{2}-\sum_{i=1}^{r}\left(X_{i+} * X_{+i}\right)}
$$

em que,

$\mathrm{X}$ - elemento da matriz; $\mathrm{N}$ - total de observações;

i+ - somatório das linhas;

$+\mathrm{i}$ - somatório das colunas;

$\mathrm{r}$ - número de linhas da matriz.

\section{RESULTADOS E DISCUSSÃO}

Na Figura 3, estão apresentadas as imagens completas e resultantes da classificação. A partir das imagens classificadas foi calculada a matriz de erro nas quatro direções distintas ( 0 , 45, 90 e 135 graus). Nos Quadros 1, 2, 3 e 4, estão apresentadas as matrizes de erro, com os respectivos erros de omissão (EO), quando os elementos foram omitidos de sua classe correta; erros de comissão (EC), quando um elemento é assinalado a uma classe, quando deveria pertencer a outra classe, e a exatidão global (EG). Pelas figuras resultantes da classificação, nota-se que as plantas Colchão e Milho foram mais bem classificadas em comparação com Leiteiro e Timbete, para todas direções analisadas.

No Quadro 5, estão apresentados os índices kappa para as quatro direções analisadas.

$\mathrm{O}$ índice kappa informa a probabilidade de a classificação realizada ser melhor que uma classificação aleatória, enquanto a exatidão global, considerando somente os elementos corretamente classificados em relação ao número total de elementos, qualifica o descritor. Assim, os resultados foram analisados de uma forma conjunta. Verifica-se que, para as direções 45, 90 e 135 graus, apresentam razoável concordância com a planta a ser classificada, conforme Pereira (2000), com erro global de 73,44, 77,08 e 67,19\% e índice Kappa de 0,6458; 0,6944 e 0,5625 , respectivamente. A direção 0 grau, com uma exatidão global de $86,98 \%$ e índice Kappa de 0,8264 , apresentou o melhor resultado na utilização do classificador textural, sendo este classificado como de excelente concordância.

Observou-se que o tamanho da janela utilizada no algoritmo para analisar a textura influenciou o resultado da classificação. Assim, a redução de dimensionalidade, ou seja, a diminuição do número de características selecionadas da imagem contribuiu, significativamente para melhor desempenho do classificador, em termos de memória utilizada e tempo computacional. 
A escolha da amostra que caracteriza a classe também é fator importante na segmentação. Ela deve ser escolhida de forma que contenha todo o padrão da textura e não extrapole as características pertencentes à classe em questão.

De acordo com Marceau et al. (1990), 90\% da variabilidade na precisão de classificação, usando texturas, depende do tamanho da janela usado para calcular texturas, sendo que o tamanho, em pixel, depende da textura em questão para realizar uma classificação satisfatória. Verifica-se que o tamanho da janela de $70 \times 70$ pixels utilizado contribui para satisfatória classificação das plantas, uma vez que a utilização de janelas maiores contribuiria para uma classificação mais pobre. A utilização de janelas menores poderia não ter capturado o padrão particular da maioria das classes. O tamanho ideal de janela seria aquele que produz a menor variabilidade na textura.

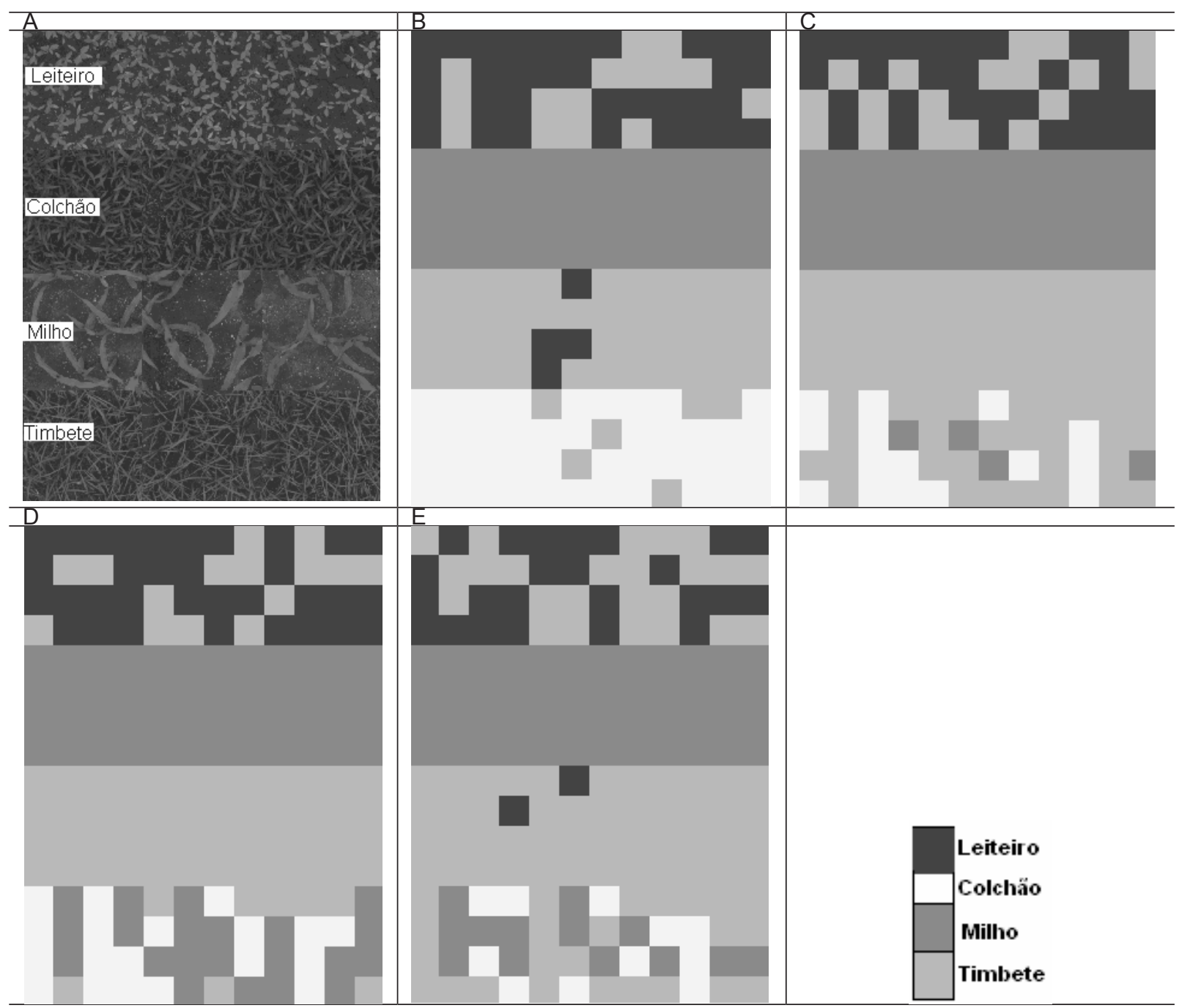

Figura 3. Ilustração das imagens e classificações: (A) Imagem Completa, classificação na direção 0 grau (B), 45 graus (C), 90 graus (D) e 135 graus (E).

Quadro 1. Matriz de erro para as quatro plantas na direção 0 grau com os erros de omissão (EO) e de comissão (EC) e a exatidão global (EG)

\begin{tabular}{lcccc|c|c}
\hline Espécies & Leiteiro & Colchão & Milho & Timbete & Totais & EO (\%) \\
\hline Leiteiro & 33 & 0 & 4 & 0 & 37 & 10,81 \\
Colchão & 0 & 48 & 0 & 0 & 48 & 0 \\
Milho & 15 & 0 & 44 & 6 & 65 & 32,31 \\
Timbete & 0 & 0 & 0 & 42 & 42 & 0 \\
\hline Totais & 48 & 48 & 48 & 48 & 192 & \\
\hline EC (\%) & 31,25 & 0 & 8,33 & 12,50 & \\
\hline
\end{tabular}


Quadro 2. Matriz de erro para as quatro plantas na direção 90 graus com os erros de omissão (EO) e de comissão (EC) e a exatidão global (EG)

\begin{tabular}{lcccc|c|c}
\hline Espécies & Leiteiro & Colchão & Milho & Timbete & Totais & EO (\%) \\
\hline Leiteiro & 30 & 0 & 0 & 0 & 30 & 0 \\
Colchão & 0 & 48 & 0 & 4 & 52 & 7,69 \\
Milho & 18 & 0 & 48 & 29 & 95 & 49,47 \\
Timbete & 0 & 0 & 0 & 15 & 15 & 0 \\
\hline Totais & 48 & 48 & 48 & 48 & 192 & \\
\hline EC (\%) & 37,50 & 0 & 0 & 68,75 & \\
\hline
\end{tabular}

EG: 73,44

Quadro 3. Matriz de erro para as quatro plantas na direção 135 graus com os erros de omissão (EO) e de comissão (EC) e a exatidão global (EG)

\begin{tabular}{lcccc|c|c}
\hline Espécies & Leiteiro & Colchão & Milho & Timbete & Totais & EO (\%) \\
\hline Leiteiro & 33 & 0 & 0 & 0 & 33 & 0 \\
Colchão & 0 & 48 & 0 & 21 & 69 & 30,43 \\
Milho & 15 & 0 & 48 & 8 & 71 & 32,39 \\
Timbete & 0 & 0 & 0 & 19 & 19 & 0 \\
\hline Totais & 48 & 48 & 48 & 48 & 192 & \\
\hline EC (\%) & 31,25 & 0 & 0 & 60,42 & \\
\hline
\end{tabular}

EG: 77,08

Quadro 4. Índices Kappa para as quatro espécies de plantas nas quatro direções analisadas

\begin{tabular}{lcccc|c|c}
\hline Espécies & Leiteiro & Colchão & Milho & Timbete & Totais & EO (\%) \\
\hline Leiteiro & 24 & 0 & 2 & 0 & 26 & 7,69 \\
Colchão & 0 & 48 & 0 & 13 & 61 & 21,31 \\
Milho & 24 & 0 & 46 & 24 & 94 & 51,06 \\
Timbete & 0 & 0 & 0 & 11 & 11 & 0 \\
\hline Totais & 48 & 48 & 48 & 48 & 192 & \\
\hline EC (\%) & 50,00 & 0 & 4,17 & 77,08 & & \\
\hline
\end{tabular}

EG: 67,19

Quadro 5. Índices Kappa para as quatro espécies de plantas nas quatro direções analisadas

\begin{tabular}{cc}
\hline Direção & Kappa \\
\hline 0 grau & 0,8264 \\
45 graus & 0,6458 \\
90 graus & 0,6944 \\
135 graus & 0,5625 \\
\hline
\end{tabular}

\section{CONCLUSÕES}

- A classificação textural para identificação de plantas é uma das mais simples possíveis, sendo os resultados das classificações extremamente relacionados com as texturas analisadas.

- Os resultados demonstraram que a abordagem é bastante promissora, podendo ser aplicada a uma grande variedade de imagens.

- A relação entre o tamanho da janela utilizada e a resposta produzida mostrou-se bastante satisfatória, obtendo-se exatidão global de 86,$98 ; 73,44 ; 77,08$ e $67,19 \%$ e índice Kappa de 0,8264; 0,6458; 0,6944 e 0,5625, para as direções $0,45,90$ e 135 graus, respectivamente. 


\section{REFERÊNCIAS}

AKSOY, S. Textural features for image database retrieval. Dissertação (Mestrado) — University of Washington, Seattle, WA.1998.

AYALA, W.; DOMINGO, J. Spatial size distributions: Applications to shape and texture analysis. IEEE Transactions on Pattern Analysis and Machine Intelligence, Spain, v.23, n.12, p.1430-1442, 2001.

BISHOP, Y.; FIENBERG, S.; HOLLAND, P. Discrete multivariate analysis: theory and practice. Cambridge, Estados Unidos: MIT, 1975. 575p.

CARD, D. H. Using know map category marginal frequencies to improve estimates of thematic map accuracy. Photogrammetric Engineering and Remote Sensing, v.43, n.3, p.431-439, 1982

CONGALTON, R. G; GREEN, K. A practical look at source of confusion in error matrix generation. Photogrammetric Engineering and Remote Sensing, v. 59, n. 5, p. 641-644, 1993.

CONNERS R. W.; HARLOW C. A. A. Theoretical Comparison of Texture Alogrithms, IEEE Transactions on Pattern Analysis and Machine Intelligence, v.2, n.3, p.204-222, 1980

DUDA, R; HART, P. Pattern Classification and Scene Analysis. New York: John \& Sons, 1973. $482 p$.

FERREIRA, M. A. L; FIGUEIREDO, A. M. Chroma Key a partir de imagens de TV Utilizando análise de Textura. Pontifícia Universidade Católica do Rio de Janeiro, Departamento de Informática 6p. 2006. Disponível em: <www.tecgraf.puc-rio.br/ mgattass/ fcg/trb06/T2-Aurelio\%20\&\%20Mauricio/paper1. doc>. Acessado em 18 out de 2006.

HARALICK,R.M.;SHANMUGAM,K.;DINSTEIN, I. Textural features for image classification. IEEE Transactions on Systems, Man and Cybernetics, v.3, n.6, p.610-621, 1973.

IIVARINEN, J.; VISA, A. An adaptive texture and shape based defect classification. In: In Proc. International Conf. on Pattern Recognition. [S.1.; s.n.], 1998. p. 117-123.
LI, S.Z. Markov Random Field, Modeling in Computer Vision, Springer-Verlag, ISBN 0-387-70145-1, 1995

LIEW, S. C. et al. Texture analysis of SAR images. In: International Geoscience and Remote Sensing Symposium. [S.1.; s.n.], 1995. p.1412-1414.

MARCEAU, D. J. et al. Evaluation of the grey-level coocurrence matrix method for land-cover classification using SPOT imagery. IEEE Transactions on Computers, Waterloo, Canada, p.513-519, 1990.

MUDIGONDA, N. R.; RANGAYYAN, R. M.; DESAUTELS, J. E. L. Gradient and texture analysis for the classification of mammographic masses. IEEE Transactions on Medical Imaging, v.19, n.10, p.1032-1043, 2000.

PEREIRA, A. K.; OLIVEIRA, E. A.; LEITE, H. V.; CABRAL, A. C. V. Correlação entre o Diagnóstico Morfológico Pré e Pós-natal das Nefrouropatias Fetais. Revista Brasileira de Ginecologia e Obstetrícia v.22, n.6, p.365-371, 2000.

PIERCE, L. et al. Texture features for classification with ERS/JERS composites. IEEE Transactions on Computers, p.348-350, 1998.

ROSENFIELD, G. H.; FITZPATRICK, K. A coefficient of agreement as a measure of thematic classification accuracy. Photogrammetric Engineering \& Remote Sensing, v.52, n.2, 1986. p.233-227.

SCHOWENGERDT, R. A. Techniques for Image Processing an Classification in Remote Sensing. Arizona: Academic Press, 2000. 520 p.

SHABAN, M. A.; DIKSHIT, O. Textural classification of high resolution digital satellite imagery. IEEE Transactions on Computers, p. 2590-2592, 1998.

SOLBERG, A. H. S.; JAIN, A. K. A study of the invariance properties of textural features in SAR images. IEEE Transactions on Computers, p.670-672, 1995.

STORY, M. Accuracy assessment: user's perspective. Photogammetric Engineering and Remote Sensing, v.52, n.3, 1986. p.397-399.

TURCEYAN, M and A. K. JAIN. Handbook of Pattern Recognition and Computer Vision, chapter Texture Analysis, p. 235-276. World Scientific Publishing Company, 1993. 